\title{
Review
}

Psychopathology

Psychopathology 2018;51:295-305

DOI: $10.1159 / 000492620$
Received: March 8, 2018

Accepted after revision: August 2, 2018 Published online: September 5, 2018

\section{Cognitive Impairment Along the Course of Depression: Non-Pharmacological Treatment Options}

\author{
Lena Listunova $^{a}$ Corinna Roth $^{a}$ Marina Bartolovic ${ }^{a}$ Johanna Kienzle ${ }^{a}$ \\ Claudia Bach $^{\mathrm{a}}$ Matthias Weisbrod ${ }^{\mathrm{a}, \mathrm{b}}$ Daniela Roesch-Ely ${ }^{\mathrm{a}}$ \\ ${ }^{a}$ Department of General Adult Psychiatry, Center for Psychosocial Medicine, Division Neurocognition,

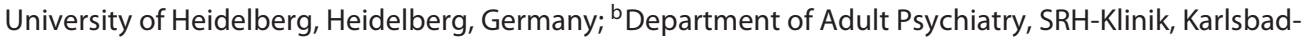 \\ Langensteinbach, Germany
}

\section{Keywords \\ Cognition disorders - Cognitive remediation - Depressive disorder - Review - Physical exercise - Yoga - Mindfulness- based therapy $\cdot$ Neuromodulation approach}

\begin{abstract}
Major Depressive Disorder (MDD) is one of the most common psychiatric disorders, with a large global impact on both the individual and the society. In this narrative review, we summarize neurocognitive deficits during acute and (partially) remitted states of depression. Furthermore, we outline the potential negative effect of cognitive impairment $(\mathrm{Cl})$ on functional recovery, and discuss the role of several variables in the development of $\mathrm{Cl}$ for MDD patients. Though there is cumulating evidence regarding persistent $\mathrm{Cl}$ in unipolar depression, research on treatment options specific for this patient group is still scarce. Hence the central aim of our review is to present non-pharmacological interventions, which are thought to reduce $\mathrm{Cl}$ in affected MDD patients. We discuss cognitive remediation therapy (CRT), physical exercise, yoga, mindfulness-based therapy, and modern neuromodulation approaches like neurostimulation and neurofeedback training. In conclusion, we propose future directions for research on $\mathrm{Cl}$ in depression. Looking
\end{abstract}

further ahead, we suggest creative interventional designs that include a direct comparison of different non-pharmacological treatment approaches on neurocognition and functional outcome of MDD. Furthermore, additive and synergistic effects of CRT with other treatment approaches should be examined and compared to create multimodal and even personalized intervention programs.

(c) 2018 S. Karger AG, Basel

\section{Introduction}

Major Depressive Disorder (MDD) is one of the most common psychiatric disorders with an estimated lifetime prevalence of $16 \%$ [1]. MDD, however, is not solely characterized by its high prevalence but also by its major global impact. In addition to substantial personal consequences of depressive symptomatology, the direct and indirect burden of the disease are associated with considerable socioeconomic costs [2]. Thus, an examination and identification of factors associated with the course of illness and its functional outcome helps in designing treatment interventions, which not only reduce the individual burden but also, in consequence, the entire socioeconomic system.

\section{KARGER}

(C) 2018 S. Karger AG, Basel

E-Mail karger@karger.com

www.karger.com/psp
Lena Listunova

Department of General Adult Psychiatry, Center for Psychosocial Medicine

Division Neurocognition, University Clinic Heidelberg

Vossstrasse 4, DE-69115 Heidelberg (Germany)

E-Mail olena.listunova@ med.uni-heidelberg.de 
Cognitive impairment (CI) during depression was identified as one of the factors predicting the relapse or recurrence of MDD [3]. CI, in particular, has emerged as a meaningful predictor of the functional outcome of depression [4]. The longitudinal examination of CI shows that various measurable deficits are found in the acute and remitted states of depression, despite pharmacological and psychotherapy interventions [5].

\section{Cl Over the Course of Depression}

CI is estimated to occur in around two-thirds of acutely depressed patients [6] and is already present in firstepisode depression [7]. Although a consistent neuropsychological pattern of deficits remains to be determined [8], empirical evidence of impaired cognitive capabilities is regularly found in several domains, that is, psychomotor function [7, 9-12], attention [6-8], memory and learning $[6-8,13,14]$, and executive functioning $[6,7,10$, $15,16]$.

In the remission phase of depression, there is evidence that CI may persist [17]. Therefore, a distinction is made between CI as state-marker (which correlates with mood symptoms and diminishes with recovery) as opposed to persistent $\mathrm{CI}$, which rather could be interpreted as traitmarker of the disease [7]. To evaluate the effect of CI along the course of depression, research is generally conducted in states with varying degrees of depression severity [18-20]. This approach is based on the assumption that a potential association between mood state and cognitive abilities exist, in which improved cognitive performance is found during states of reduced depression severity compared to clinically relevant states. Existing data indicate persisting and significant CI, despite partial remission of depressive symptomatology [6].

It is estimated that CI affects one-third to one-half of previously depressed subjects [6, 21-23]. Moreover, a study by Bhalla et al. [23] revealed that CI persists in an approximate $94 \%$ of remitted patients who displayed cognitive difficulties during clinical late-life depression.

Similar to the acute state, a specific pattern of deficits in remission remains to be determined, though evidence of CI is regularly found in domains also emphasized in the context of acute depression, that is, psychomotor function $[19,24,25]$, attention $[6,8,20,26]$, memory and learning $[6,8,19,27,28]$, and executive function $[6,18$, $19,26,28-30]$.

All in all, empirical findings regarding CI in remitted subjects are not conclusive. Improvement of the se- verity of symptomatology seems to positively impact several neuropsychological processes and the overall cognitive functioning of subjects. Yet, these effects appear to be differential for each domain, with psychomotor functioning and memory showing a stronger association with the mood state than attention and executive function, which tend to persist independent of mood improvement. Consequently, research suggests that CI cannot be completely accounted for by the severity of depressive symptomatology. These results reflect a great uncertainty regarding CI in remitted depression and highlight the importance of further research.

\section{Impact of $\mathrm{Cl}$ on Functional Recovery}

Persistent CI is being discussed as a potential cause for higher functional impairment in MDD patients. CI appears to significantly contribute to the observed poor functional outcome in depression [4, 31]. Longitudinal research by Jaeger et al. [4] linked persistent cognitive deficits in MDD patients at a 6-month followup after hospitalization to a poorer functional outcome, with the severity of CI being predictive of the level of recovery. This association remained significant when controlling for residual depressive symptoms, indicating that $\mathrm{CI}$ independently impacts functional recovery.

CI was also found to be associated with poor response to antidepressant treatment $[32,33]$ in late-life depression as well as an unfavorable outcome of Cognitive Behavioral Therapy [34]. Furthermore, MDD patients with neuropsychological deficits experience higher rates of relapse and recurrence [3]. Finally, CI has been shown to be a principal mediator of psychosocial impairment, notably workforce performance, in MDD [35].

\section{Variables Associated with $\mathrm{Cl}$ in MDD}

As described in the previous section, empirical evidence suggests that CI might be an important factor contributing to poorer functional outcome in MDD, and consequently may be referred to as clinically relevant. In consequence, the examination of variables associated with persisting CI in MDD might help to better understand underlying mechanisms as well as identify possible risk groups regarding the development of $\mathrm{CI}$ in the course of illness. 
A number of variables associated with CI have been thoroughly analyzed and described in literature, for example, various demographic variables like age $[17,36]$, educational attainment [7, 37, 38], and employment status [8]. Also, variations concerning disease variables and psychopathology, like onset of disorder [17, 36, 3941], duration of illness [42], number of past hospitalizations $[17,43]$, and current symptom severity $[20,24$, 28 ] have been found to be associated with the development of CI and the degree to which it presents itself in MDD patients. Some of the inconsistencies in literature regarding the prevalence and profile of $\mathrm{CI}$ in depression might be due to complex interactions between these variables.

\section{Age, Onset and Duration of Disorder}

It is commonly accepted that geriatric MDD patients are cognitively more impaired than younger subjects [17, 36], due to several somatic risk factors promoting CI [39]. In particular, the impact of vascular lesions has been well established and may account specifically for the persistent CI found among elderly subjects with late onset of depressive disorder [17, 36, 39, 41]. Some authors also described important differences in CI when comparing geriatric early- vs. late-onset MDD patients, in that those with late onset showed stronger impairments in processing speed and executive function than patients with early onset, while both patient groups showed reduced function in all domains compared to healthy controls [40]. Closely related to age at onset is the duration of illness referring to how much time has passed since onset. A longer duration has been linked to poorer cognitive performances [42].

\section{Educational Attainment}

Previous research has found education to positively impact cognitive functioning in MDD patients $[7,37,38]$. However, protective effects of educational attainment were mainly examined in older individuals. A meta-analysis by Lee et al. [7] that extended the age-range to samples with younger depressive subjects, found less educated samples to demonstrate more pronounced CI in several domains (visual and verbal memory as well as attentional switching), supporting the protective relevance of educational effects in non-geriatric patients.

\section{Employment Status}

The rate of depressed subjects among the unemployed appears to be significantly increased when compared to the employed population [44]. It is hypothesized that cognitive functioning might be an important factor in the relationship between employment status and depression [8]. Unemployment was shown to be significantly associated with poorer cognitive functioning (memory, attention, and executive function), particularly in remitted MDD patients, with those unemployed performing worse than those employed [8]. The direction of this effect remains unclear due a lack of prospective studies: CI can be interpreted either as cause or consequence of unemployment, or possibly both.

\section{Hospitalization}

When separating acute MDD samples by current hospitalization, studies consistently demonstrate more pronounced CI (including psychomotor speed, working memory, as well as verbal and visual memory) in inpatients compared to outpatients, maybe linked to a more severe acute symptomatology and/or to a higher prevalence of the endogenous/melancholic subtype of depression $[43,45]$. Furthermore, a history and higher number of hospitalizations have also been found to be associated with more severe CI [43]. The number of past hospitalizations might be an indicator of illness chronicity and severity, which in turn maybe linked to more pronounced CI.

\section{Residual Symptomatology}

Depressive symptomatology has been shown to relate to the overall neuropsychological functioning [20]. The majority of findings, however, are based on clinically depressed patients in comparison to those in remission or healthy controls, ignoring the question of how subclinical psychopathology might affect persisting CI. Though there appears to be an overall tendency towards cognitive improvement parallel to symptom remission, the level of subclinical severity has been shown to impact neuropsychological performances (e.g., memory, psychomotor function) $[24,28]$, indicating the importance of considering residual depressive symptomatology in examining persistent CI.

\section{Treatment Approaches}

Since cognitive deficits in MDD may persist beyond routine treatment of depressive symptoms, evaluation of specific treatment options is of critical importance. There is a growing number of studies and reviews concerning the impact of pharmacological strategies on neurocognitive functioning [46-49]. There is limited 
evidence for the pro-cognitive effects of conventional antidepressants, also due to lack of clinical trials targeting cognition as a primary outcome. A recent metaanalyses [47] of nine placebo-controlled randomized trials evaluated the cognitive effects of vortioxetine, duloxetine, paroxetine, citalopram, phenelzine, nortryptyline, and sertraline and showed low effect sizes for a positive effect of antidepressants on processing speed (standard mean difference 0.16$)$ and delay recall (0.24) [47]. Among evaluated antidepressant, vortioxetine appeared to have the largest effect size on psychomotor speed (0.34), cognitive control (0.17), and executive function (0.26), while duloxetine had the greatest effect on delayed recall (0.25) [47]. One of the studies in this meta-analysis evaluated the efficacy of vortioxetine on cognitive function in adults with recurrent moderateto-severe MDD [48]. The study showed a significant improvement of objective and subjective measures of cognitive functions with vortioxetine (standard effect sizes $0.23-0.52$ ), which was largely independent of vortioxetine's effect on improving depressive symptoms [48]. Furthermore, the neurocognitive aspects of $\mathrm{N}$ methyl-D-aspartate glutamate receptor antagonist ketamine was evaluated in addition to its rapid antidepressant effects in treatment-resistant depression [49]. The authors showed a significant improvement in scores of visual memory, simple and complex working memory after the sixth ketamine infusion. However, neurocognitive changes were accounted for by improvement in the depressive severity. It remains uncertain if cognitive improvement was mainly attributed to the improvement of depressive symptomatology and if persistent CI in/after the remission can be improved. Moreover, baseline neurocognition seemed to predict ketamine response.

Beside the pro-cognitive effects, side effects of antidepressant therapies on cognition have to be considered [50], including anticholinergic effects of tricyclic antidepressants $[51,52]$. It is important to mention that responders and non-responders to SSRIs could already be distinguished in neuropsychological terms before treatment, with non-responders showing a higher degree of CI [53]. According to Bortolato et al. [46], beside conventional treatment with antidepressants, there are promising pro-cognitive agents like Erythropoietin, S-adenosyl methionine, insulin, $\mathrm{N}$-acetylcysteine, and antidiabetic agents to be further investigated [35].

In all, the cognitive improvement remains an unmet need in the pharmacological treatment of CI. Furthermore, the side effects should be taken into consideration.
Thus, non-pharmacological interventions focusing on CI may be valuable as a treatment element for affected MDD patients. Our review focuses on the growing but still limited body of non-pharmacological treatment approaches. In the following sections, we discuss cognitive remediation therapy (CRT), physical exercise, mind-body Interventions like yoga and mindfulness-based therapies, and neuromodulation approaches.

\section{Literature Search}

Studies until April 2017 were identified by searching PubMed and PsychInfo databases, using the following search terms in different combinations: "depression", "depressive disorder", "MDD", "cognition", "cognitive training", "cognitive remediation", "improve cognition", "neurocognitive training", "computer-based", "nonpharmacological interventions", "physical exercise", "physical intervention", “yoga”, "meditation", "mindfulness-based", "neuromodulation", "neurostimulation", "neurofeedback", "repetitive transcranial magnetic stimulation (rTMS)", "transcranial direct current stimulation (tDCS)". Only studies published in English were considered for this review. After selecting pertinent systematic reviews and meta-analysis, the full text of the studies included in these meta-analyses was reviewed. Outcome measures included global cognition and different cognitive domains (speed of processing, attention, working memory, verbal and visual memory, and executive functioning). The results were limited to participants aged 18 years or more. Reference lists of relevant articles, previous reviews, and Google scholar were examined to identify further relevant articles.

\section{Cognitive Remediation Therapy}

One of the regularly discussed treatments addressing $\mathrm{CI}$ is CRT. CRT in general involves two different training approaches: direct restitution of impaired cognitive functions using heterogeneous paper-pencil or computerized cognitive training (CCT) techniques vs. strategy training which rather aims at compensating everyday difficulties associated with CI. However, nowadays most of the programs include both approaches: direct "drill and practice" of cognitive functions in combination with compensatory strategy learning, psycho-education on cognition-related issues, and/or transfer to real-world situations [54]. 
Up to now, merely a few publications have examined the effect of CRT in affective disorder samples, whereby some included exclusively bipolar $[55,56]$ or unipolar patients [57-65], while others investigated both groups together $[66,67]$.

A recent meta-analysis by Motter et al. [68] evaluated the efficacy of CCT in MDD and included nine randomized controlled CCT trials (in one study, patients with a diagnosis of dysthymia were included [63]). The investigation revealed CCT to be associated with improved depressive symptomatology and everyday functioning (significant small to moderate effects). The examination yielded moderate to large effects for attention (Hedge's $\mathrm{g}=0.67$ ), working memory ( $\mathrm{g}=$ $0.72)$, and global functioning $(\mathrm{g}=1.05)$ on the one hand and small and non-significant effects regarding executive functioning $(\mathrm{g}=0.20)$ and verbal memory $(\mathrm{g}=$ 0.08 ) on the other hand. Furthermore, slightly lower effects of CCT on age were found. The lack of significant effects for those two domains could either be attributed to methodological issues like highly heterogeneous measures or insufficient assessment. In fact, executive functioning was only targeted in one study during the last 2 weeks of training [57] and only one session of psycho-education in another [62]. However, it could also reflect valid difficulties in improving those two cognitive domains, which need to be theoretically discussed and taken into account when designing specific training approaches.

Regarding the effects on everyday functioning, it must be taken into account that only two studies included in the meta-analysis assessed appropriate transfer measures $[57,62]$. Therefore, more studies assessing the effect of the CCT on everyday functioning are needed to confirm the positive findings in this meta-analysis.

However, most of the included studies investigated acute MDD patient groups. Only two clinical trials of this meta-analysis examined the impact of CCT on stable patients with MDD without psychotic symptoms [59, 62]: Elgamal et al. [59], for example, demonstrated improvement under CRT in several neuropsychological tests regarding attention, verbal learning and memory, psychomotor speed, and executive function in $n=12$ outpatients with long-term illness with stable depressive symptom scores over a time period of ten weeks. Naismith et al. [62] investigated $n=41$ stabilized outpatients on medication with a lifetime history of major depression and depressive symptoms in the normal to mild range. Patients with the mean age of 64.8 years were randomized either to a waitlist or to an intervention group. The intervention group underwent a "neuropsychological educational approach to remediation" (NEAR) and multifactorial psycho-education that targeted cognitive strategies, depression, anxiety, sleep, vascular risk factors, diet, and exercise. After 10 weeks of training, patients showed significant improvements in visual and verbal memory (medium to large effect sizes).

Despite the first promising effects of CRT on cognition and functional outcome shown in the meta-analysis, the optimal design of CRT interventions remains a matter of debate. The training designs implemented in the included studies varied substantially, making it impossible to infer general statements or even recommendations regarding the optimal techniques, frequency or duration of CRT. For example, besides using different training software, the duration of CCT intervention among the studies ranged from 5 days [64] to 16 weeks [61]. The training intensity also varied from 3 sessions over 2 weeks [58] to 64 sessions over 16 weeks [61]. Furthermore, only two of the included studies combined CCT with compensatory strategies $[57,62]$. Naismith et al. [62] provided the intervention group with a psychoeducation training on health and cognitive functioning as already mentioned above, over a period of 10 weeks. Bowie et al. [57], however, supplemented the CCT with strategic self-monitoring training, bridging discussions to facilitate transfer to everyday behavior and 2 homework sessions per day, also over the period of 10 weeks. Thirty-three participants with a diagnosis of MDD and treatment resistance were allocated, either to the intervention or wait list group. The study revealed treatment effects on verbal memory, attention, and processing speed, but not on executive functioning. Although no changes in everyday functional skills and behavior were observed in the intervention group, a significant modest correlation of cognitive improvements with improvements in the ratings of everyday functional behaviors was found.

Several authors have also emphasized the need to individualize CRT to increase the effects on cognition, motivation, and transfer to real-world situations [54, 69]. This approach contrasts with generalized training programs, which target a broad set of functions in all patients. Our research group currently examines the efficacy of generalized vs. individualized cognitive training (manuscript in preparation), among other important questions. To summarize, there is preliminary evidence for a beneficial effect of CRT on patients with MDD. For future research and clinical practice, there is still need to determine the optimal intensity and frequency, duration and 
combination with additional valuable psychosocial treatment approaches. Moreover, the contribution of motivation or expectancy on the differential performance between intervention and control groups should be targeted in future investigations.

\section{Physical Exercise Interventions}

Physical exercise has been shown to be effective in improving the cognitive functioning in different groups like healthy subjects $[70,71]$, individuals suffering from mild CI [72], dementia [73], schizophrenia, and affective disorders [74]. There is a small number of studies, which investigated the effect of physical exercise on neurocognition in MDD. Despite equivocal and inconsistent study results, physical exercise might be a promising intervention to enhance cognition in depression [74]. Until now, physical exercise interventions were mostly investigated among healthy older adults. Physical activity as well as long-term exercise may preserve cognition and prevent or even delay dementia at least at the community level $[75,76]$. A meta-analysis of randomized controlled aerobic exercise trials examined the relationship between an aerobic exercise training of at least 1 month on neurocognitive performances in a nondement sample [71]. Twenty nine studies were included in this meta-analysis, which revealed modest improvements of attention and processing speed, executive function, and memory as well as less consistent effects on working memory. Combined aerobic exercise and strength training interventions improved attention, processing speed, and working memory more than aerobic exercise alone. Furthermore, individuals with mild CI showed a stronger enhancement of memory performance through physical exercise than cognitively unimpaired individuals [71].

A recently published systematic review and metaanalysis of Brondino et al. [77], addressing possible effects of physical exercise on cognitive functioning in depression, including eight randomized controlled trials, showed no improvement of cognition in MDD through physical exercise (one study used yoga as physical exercise intervention [78]). Neither the duration of the intervention, nor the number of sessions per week or the intensity of exercise impacted the outcome significantly. The results of this meta-analysis contradict at the first glance the comprehensive review of Malchow et al. [74], who reported positive effects of physical exercise on schizophrenia and affective disor- ders, thereby recommending physical exercise as part of a multimodal intervention program to improve neurocognition and psychopathology. However, the results of Brondino et al. [77] should be taken with caution because of several methodological limitations, already mentioned by authors: None of the included studies recruited patients with subjective cognitive complaints. Patients were only slightly cognitive impaired at baseline, and therefore had a small potential for cognitive improvement. Furthermore, improvement in neurocognition was frequently investigated as a secondary outcome and was not the main focus of investigation. Therefore, studies could be potentially statistically underpowered to find a significant difference in this regard.

In sum, the existing number of randomized controlled trials that systematically investigate the effects of physical exercise on neurocognition in MDD (especially in remission) is promising, albeit still limited and inconsistent. However, taking also into account the described positive findings regarding cognitive improvement through aerobic exercise in other investigated populations [79-81], future research into the impact of physical exercise on persistent CI in MDD is further needed.

Moreover, there is some evidence that the combination of physical exercise and cognitive training can have additive and synergistic effects on neurocognition, possibly by the following path: The potential for neurogenesis and synaptogenesis can be increased through physical exercise, while cognitive training guides it to induce positive plastic change [82]. The effects of combined cognitive and physical interventions on cognition in healthy older adults were examined in a recent metaanalysis, which included twenty studies with older adults without known CI [70]. The meta-analysis revealed greater effects of combined interventions over control group and physical exercise alone, but no significant advantage over cognitive intervention alone. Additionally, the effects were moderated by age, intervention setting, and frequency. The first study in the literature, known to us, that combined cognitive and physical exercise training across two patient groups (29 patients with schizophrenia and 22 patients with MDD) showed the strongest effects on cognitive functioning and psychopathological symptoms across patients groups in subjects undergoing combined cognitive and aerobic physical training. The domains of visual learning, processing speed, and working memory were improved in both patient groups. The combination of cognitive and physical exercise training was superior to the combination of $\operatorname{cog}$ - 
nitive training and relaxation as well as waiting control group [80]. These findings point to a possible benefit of physical exercise as an add-on therapy to other interventions regarding improvement of cognitive functioning in different patient groups. However, future replication is needed.

\section{Yoga}

Another approach to enhance neurocognitive functioning is yoga intervention, which involves an active attentional component above its physical activity component and is easily adaptable for most ages and clinical populations. Yoga requires focused effort on breathing, body, and pose control, which can lead to even greater cognitive improvement compared to physical exercise interventions without any attentional component (e.g., [83]). A recent meta-analysis on the effects of long-term yoga interventions and acute (immediate, single session) yoga practice on cognition revealed its potential to improve specific domains of neurocognitive functioning despite the limited number of studies conducted, small sample sizes, and other design weaknesses of the included studies [84]. This meta-analysis, which included 22 studies with diverse sample characteristics, including cognitively unimpaired, healthy, and clinical populations, showed moderate effects of yoga on cognition in randomized controlled trials (long-term yoga interventions), with the strongest effects for attention and processing speed, followed by executive function and memory. The meta-analysis yielded a stronger overall effect of yoga on cognition in counterbalanced repeated-measures acute exposure studies (sessions that lasted between 9 rounds of breathing exercises up to $45 \mathrm{~min}$ ) than in long-term yoga interventions (ranged from 1 to 6 months in duration). The strongest effect was on memory, followed by attention, processing speed, and executive functioning [84]. Only one study included in the meta-analysis investigated the effect of yoga on neurocognition in a depressive sample. Sharma et al. [78] found an additional improvement in some measures of executive functions in (partially) remitted depressive patients, who were randomly allocated to the yoga meditation group and received conventional antidepressant medication in comparison to the group, which only received conventional antidepressant medication. All in all, although promising there is still research lacking on the efficacy of physical exercises and yoga in MDD patients.

CI in MDD: Treatment Options

\section{Mindfulness-Based Therapy}

As mindfulness is closely related to attentional processes, mindfulness-based therapy is considered as an attention training, and therefore another possible treatment approach of CI in depression. Despite the multitude of studies examining the clinical benefits of mindfulness-based therapy, research on the effects of this approach on neurocognitive functioning is scarce. There are first investigations of mindfulness interventions that revealed improvement in attention and working memory in bipolar depression [85]. Chiesa et al. [86] investigated, in a systematic review of 23 studies, the effects of mindfulness meditation practices on cognitive abilities in healthy, chronic pain, major depression, military personnel, and traumatic brain injury populations. This review revealed an enhancement of working memory capacity and some executive functions. Furthermore, the early stages of mindfulness training were associated with significant improvement of selective and executive attention, whereas the following stages improved unfocused sustained attention abilities. However, these results should be considered with caution because of methodological limitations of the studies included. There is also evidence that mindfulness meditation practices can provide benefits on cognitive abilities, in particular attention, in different psychiatric disorders like Borderline Personality Disorder, MDD, alcohol abuse, and ADHD [87-90]. The purpose of future investigation and clinical practice is to create and modify mindfulness-based therapies for specific psychiatric and neurological disorders. Mindfulness-Based Cognitive Therapy ([91]) and Mindfulness-Based Stress Reduction ([92]) are, for example, already widely incorporated into the treatment of affective disorders but barely examined in their ability to improve cognitive functioning. Taking into account the promising preliminary results of cognitive enhancement in different populations due to mindfulness-based therapy, high quality studies investigating standardized mindfulnessbased approaches and comparing them to other interventions are needed.

\section{Neuromodulation Techniques: Neurostimulation and Neurofeedback Training}

Novel neuromodulation approaches can also be considered as treatment options of CI in MDD. One possible technique, which evolved in recent years is the non-inva- 
sive stimulation of specific brain regions. Especially, brain activity in the dorsolateral prefrontal cortex (DLFPC) areas has been identified as a possible goal for such techniques, trying to enhance associated working memory performances. A systematic review and meta-analysis of non-invasive brain stimulation techniques included 12 studies that used either repetitive rTMS or tDCS to increase DLFPC activity, and therefore working memory performance in healthy and clinical samples [93]. rTMS showed significant improvement of all measures of working memory performance, whereas tDCS improved only the response time. A study by Wolkenstein et al. [94] revealed that anodal tDCS to the DLFPC enhanced working memory performance in $22 \mathrm{MDD}$ patients and 22 healthy controls [94].

Another treatment approach, neurofeedback, aims at teaching patients to self-regulate their brain activity with the help of real-time feedback to achieve specific goals. One possible goal is enhancement of neurocognitive performances. A first study comparing the cognitive performances of a neurofeedback training group to a non-interventional control group in a sample of MDD patients $(n=$ 60 ) showed significant improvement in working memory and processing speed with medium to large effect sizes [95]. However, there are some limitations that need to be considered, like the lacking of randomization and mixed depressive states of patients. In sum, future research is required.

\section{Future Directions}

Evidence shows that one-third to one-half of depressive patients continue suffering from CI even into remission. Research on the underlying factors causing and maintaining $\mathrm{CI}$ in MDD is recent but growing considerably. Up to now, it is still not clear for how long CI may persist, and a clear pattern of cognitive deficits could not yet be elucidated. Various demographic and psychopathological characteristics are being investigated in relation to the level of cognitive functioning during depression. However, a more comprehensive understanding of relevant factors is still lacking. Preliminary research on CI associated variables suggest that treatment of CI in MDD patients might be useful early on, namely when a patient shows cognitive risk factors. As the number of past hospitalizations is associated with more severe CI, an initiation of cognitive treatment immediately at first-time hospitalization would be beneficial for the patients. It may also be beneficial to provide additional compensatory strategy for learning and psycho-education on cognition-related issues offering background information, especially for less educated patients. Furthermore, the different possible cognitive profiles of patients with late-onset of depressive disorder (stronger impairments in processing speed and executive function than patients with early onset) should be investigated, to provide tailored interventions to this group of patients. CI can be interpreted either as a cause or consequence of unemployment, or possibly both. Home-based cognitive treatment could be useful for employed (partially) remitted patients with MDD, and persisting CI for better compatibility with work responsibilities. Research on the impact of persistent CI on general everyday functioning and clinical aspects in MDD patients is currently emerging and is a promising field.

There is some evidence regarding the treatment of $\mathrm{CI}$ in MDD, such as in the field of schizophrenia, for positive CRT effects on cognition and functional outcomes. Interventional studies evaluating the effect of other specific treatment approaches for persistent cognitive deficits and its impact on functional outcome are of growing interest. Promising findings of cognitive improvement through physical exercise in healthy elderly populations, individuals suffering from mild CI, dementia, schizophrenia and affective disorders, and through yoga in healthy and clinical cognitively unimpaired populations already exist. However, research on the efficacy of physical exercise and yoga in (subjectively) cognitively impaired (remitted) depressive populations is still lacking. Novel investigations of the mindfulness-based therapy and neuromodulation techniques like neurostimulation (rTMS, tDCS) and neurofeedback training also show positive results in healthy as well as clinical populations and require further research in the sample of MDD patients.

There is also a need to provide a consensus on the appropriate screening, measurement, and monitoring tools. Due to the high level of heterogeneity of neurocognitive testing, comparing cognitive clinical trials becomes complex. Thus, standardization of cognitive testing is required. In Schizophrenia research, this has already been accomplished with the MATRICS Battery [96]. A combination of both self-report and objective cognitive testing may lead to a better understanding of subjective complaints in MDD [47].

Looking further ahead, creative interventional designs may include a direct comparison of different non-pharmacological treatment approaches on neurocognitive 
functioning and functional outcome of MDD. Furthermore, additive and synergistic effects of CRT with other treatment approaches (physical exercise, yoga, mindfulness-based therapy, and neuromodulation techniques) should be examined and compared to create multimodal and even personalized intervention programs to improve neurocognition and psychosocial functioning of patients with MDD. In our opinion, a combination of different interventions, for example, of physical exercise with subsequent cognitive training sessions may be promising as well as superior to a single-intervention design. Reasons behind this assumption are neuroplasticity effects of physical exercise serving as a basis for improved learning through cognitive remediation. Furthermore, various treatment options should be considered, and cognitive treatment customized to patients' needs and wishes while considering available resources (e.g., computer access) or physical functioning.

\section{Acknowledgements}

This study was funded by the DFG (project number: RO 3418/6-1).

\section{Disclosure Statement}

D.R.-E. and M.W. have contracts for the development of neuropsychological diagnostic and training tools with Schuhfried $\mathrm{GmbH}$. The other authors report no conflicts of interest.

\section{References}

1 Kessler RC, Berglund P, Demler O, Jin R, Koretz D, Merikangas KR, et al: The epidemiology of major depressive disorder: results from the national comorbidity survey replication (NCS-R). Jama 2003;289:3095-3105.

2 Whiteford HA, Ferrari AJ, Degenhardt L, Feigin $\mathrm{V}$, Vos T: The global burden of mental, neurological and substance use disorders: an analysis from the global burden of disease study 2010. PLoS One 2015;10:e0116820.

3 Majer M, Ising M, Künzel H, Binder E, Holsboer F, Modell S, et al: Impaired divided attention predicts delayed response and risk to relapse in subjects with depressive disorders. Psychol Med 2004;34:1453-1463.

4 Jaeger J, Berns S, Uzelac S, Davis-Conway S: Neurocognitive deficits and disability in major depressive disorder. Psychiatry Res 2006; 145:39-48.

5 Douglas KM, Porter RJ: Longitudinal assessment of neuropsychological function in major depression. Aust N Z J Psychiatry 2009;43: 1105-1117.

6 Rock P, Roiser J, Riedel W, Blackwell A: Cognitive impairment in depression: a systematic review and meta-analysis. Psychol Med 2014; 44:2029-2040.

7 Lee RS, Hermens DF, Porter MA, RedobladoHodge MA: A meta-analysis of cognitive deficits in first-episode major depressive disorder. J Affect Disord 2012;140:113-124.

8 Baune BT, Miller R, McAfoose J, Johnson M, Quirk F, Mitchell D: The role of cognitive impairment in general functioning in major depression. Psychiatry Res 2010;176:183-189.

9 Caligiuri MP, Ellwanger J: Motor and cognitive aspects of motor retardation in depression. J Affect Disord 2000;57:83-93.

10 McDermott LM, Ebmeier KP: A meta-analysis of depression severity and cognitive function. J Affect Disord 2009;119:1-8.
11 Tsourtos G, Thompson J, Stough C: Evidence of an early information processing speed deficit in unipolar major depression. Psychol Med 2002;32:259-265.

12 Zakzanis K, Leach L, Kaplan E: On the nature and pattern of neurocognitive function in major depressive disorder. Cogn Behav Neurol 1998;11:111-119.

13 Austin M-P, Mitchell P, Wilhelm K, Parker G, Hickie I, Brodaty $\mathrm{H}$, et al: Cognitive function in depression: a distinct pattern of frontal impairment in melancholia? Psychol Med 1999; 29:73-85.

14 MacQueen G, Galway T, Hay J, Young L, Joffe R: Recollection memory deficits in patients with major depressive disorder predicted by past depressions but not current mood state or treatment status. Psychol Med 2002;32: 251-258.

15 Elliott R: The neuropsychological profile in unipolar depression. Trends Cogn Sci 1998;2: 447-454.

16 Veiel HO: A preliminary profile of neuropsychological deficits associated with major depression. J Clin Exp Neuropsychol 1997;19: 587-603.

17 Hasselbalch BJ, Knorr U, Kessing LV: Cognitive impairment in the remitted state of unipolar depressive disorder: a systematic review. J Affect Disord 2011;134:20-31.

18 Douglas KM, Porter RJ, Knight RG, Maruff P: Neuropsychological changes and treatment response in severe depression. Br J Psychiatry 2011;198:115-122.

19 Reppermund S, Zihl J, Lucae S, Horstmann S, Kloiber S, Holsboer F, et al: Persistent cognitive impairment in depression: the role of psychopathology and altered hypothalamic-pituitary-adrenocortical (HPA) system regulation. Biol Psychiatry 2007;62: 400-406.
20 Roca M, López-Navarro E, Monzón S, Vives M, García-Toro M, García-Campayo J, et al: Cognitive impairment in remitted and nonremitted depressive patients: a follow-up comparison between first and recurrent episodes. Eur Neuropsychopharmacol 2015;25: 1991-1998.

21 Abas MA, Sahakian BJ, Levy R: Neuropsychological deficits and CT scan changes in elderly depressives. Psychol Med 1990;20: 507-520.

22 Reppermund S, Ising M, Lucae S, Zihl J: Cognitive impairment in unipolar depression is persistent and non-specific: further evidence for the final common pathway disorder hypothesis. Psychol Med 2009;39:603-614.

23 Bhalla RK, Butters MA, Mulsant BH, Begley AE, Zmuda MD, Schoderbek B, et al: Persistence of neuropsychologic deficits in the remitted state of late-life depression. Am J Geriatr Psychiatry 2006; 14:419-427.

24 Weiland-Fiedler P, Erickson K, Waldeck T, Luckenbaugh DA, Pike D, Bonne O, et al: Evidence for continuing neuropsychological impairments in depression. J Affect Disord 2004;82:253-258.

25 Yuan Y, Zhang Z, Bai F, Yu H, Shi Y, Qian Y, et al: Abnormal neural activity in the patients with remitted geriatric depression: a restingstate functional magnetic resonance imaging study. J Affect Disord 2008;111:145-152.

26 Paelecke-Habermann Y, Pohl J, Leplow B: Attention and executive functions in remitted major depression patients. J Affect Disord 2005;89:125-135.

27 Neu P, Bajbouj M, Schilling A, Godemann F, Berman RM, Schlattmann P: Cognitive function over the treatment course of depression in middle-aged patients: correlation with brain MRI signal hyperintensities. J Psychiatr Res 2005;39:129-135. 
28 Preiss M, Kucerova H, Lukavsky J, Stepankova $H$, Sos P, Kawaciukova R: Cognitive deficits in the euthymic phase of unipolar depression. Psychiatry Res 2009;169:235-239.

29 Nakano Y, Baba H, Maeshima H, Kitajima A, Sakai Y, Baba K, et al: Executive dysfunction in medicated, remitted state of major depression. J Affect Disord 2008;111:46-51.

30 Schmid M, Strand M, Årdal G, Lund A, Hammar Å. Prolonged impairment in inhibition and semantic fluency in a follow-up study of recurrent major depression. Arch Clin Neuropsychol 2011;26:677-686.

31 Naismith SL, Longley WA, Scott EM, Hickie IB: Disability in major depression related to self-rated and objectively-measured cognitive deficits: a preliminary study. BMC Psychiatry 2007;7:32

32 Potter GG, Kittinger JD, Ryan Wagner H, Steffens DC, Ranga Rama Krishnan K: Prefrontal neuropsychological predictors of treatment remission in late-life depression. Neuropsychopharmacology 2004;29:22662271.

33 Story TJ, Potter GG, Attix DK, Welsh-Bohmer KA, Steffens DC: Neurocognitive correlates of response to treatment in late-life depression. Am J Geriatr Psychiatry 2008; 16:752-759.

34 Crews Jr WD, Harrison DW: The neuropsychology of depression and its implications for cognitive therapy. Neuropsychol Rev 1995;5: $81-123$.

35 McIntyre RS, Cha DS, Soczynska JK, Woldeyohannes HO, Gallaugher LA, Kudlow $\mathrm{P}$, et al: Cognitive deficits and functional outcomes in major depressive disorder: determinants, substrates, and treatment interventions. Depress Anxiety 2013;30:515-527.

36 Austin MP, Mitchell P, Goodwin GM: Cognitive deficits in depression. Br J Psychiatry 2001;178:200-206.

37 Avila R, Moscoso MAA, Ribeiz S, Arrais J, Jaluul O, Bottino CM: Influence of education and depressive symptoms on cognitive function in the elderly. Int Psychogeriatr 2009;21: 560-567.

38 McLaren ME, Szymkowicz SM, Kirton JW, Dotson VM: Impact of Education on Memory Deficits in Subclinical Depression. Arch Clin Neuropsychol 2015;30:387-393.

39 Hickie I, Scott E: Late-onset depressive disorders: a preventable variant of cerebrovascular disease? Psychol Med 1998;28:1007-1013.

40 Herrmann LL, Goodwin GM, Ebmeier KP: The cognitive neuropsychology of depression in the elderly. Psychol Med 2007;37:16931702.

41 Bora E, Harrison B, Yücel M, Pantelis C: Cognitive impairment in euthymic major depressive disorder: a meta-analysis. Psychol Med 2013;43:2017.

42 Elgamal S, Denburg S, Marriott M, MacQueen G: Clinical factors that predict cognitive function in patients with major depression. Can J Psychiatry 2010;55:653-661.

43 Purcell R, Maruff P, Kyrios M, Pantelis C: Neuropsychological function in young pa- tients with unipolar major depression. Psychol Med 1997;27:1277-1285.

44 Stankunas M, Kalediene R, Starkuviene S, Kapustinskiene V: Duration of unemployment and depression: a cross-sectional survey in Lithuania. BMC Public Health 2006;6:1.

45 Stage K, Bech P, Gram L, Kragh-Sørensen P, Rosenberg C, Øhrberg S: Are in-patient depressives more often of the melancholic subtype? Acta Psychiatrica Scandinavica 1998;98: 432-436.

46 Bortolato B, Miskowiak KW, Köhler CA, Maes M, Fernandes BS, Berk M, et al: Cognitive remission: a novel objective for the treatment of major depression? BMC Med 2016; 14:9.

47 Rosenblat JD, Kakar R, McIntyre RS: The cognitive effects of antidepressants in major depressive disorder: a systematic review and meta-analysis of randomized clinical trials. Int J Neuropsychopharmacol 2015; 19:pii:pyv082.

48 McIntyre RS, Lophaven S, Olsen CK: A randomized, double-blind, placebo-controlled study of vortioxetine on cognitive function in depressed adults. Int J Neuropsychopharmacol 2014; 17:1557-1567.

49 Shiroma PR, Albott CS, Johns B, Thuras P, Wels J, Lim KO: Neurocognitive performance and serial intravenous subanesthetic ketamine in treatment-resistant depression. Int J Neuropsychopharmacol 2014;17:18051813

50 Fava M, Graves LM, Benazzi F, Scalia MJ, Iosifescu DV, Alpert JE, et al: A cross-sectional study of the prevalence of cognitive and physical symptoms during long-term antidepressant treatment. J Clin Psychiatry 2006;67: 1754-1759.

51 Peretti S, Judge R, Hindmarch I: Safety and tolerability considerations: tricyclic antidepressants vs. selective serotonin reuptake inhibitors. Acta Psychiatrica Scand suppl 2000; 403:17-25.

52 Spring B, Gelenberg AJ, Garvin R, Thompson $S$ : Amitriptyline, clovoxamine and cognitive function: a placebo-controlled comparison in depressed outpatients. Psychopharmacology 1992;108:327-332.

53 Kampf-Sherf O, Zlotogorski Z, Gilboa A, Speedie L, Lereya J, Rosca P, et al: Neuropsychological functioning in major depression and responsiveness to selective serotonin reuptake inhibitors antidepressants. J Affect Disord 2004;82:453-459.

54 Medalia A, Choi J: Cognitive remediation in schizophrenia. Neuropsychol Rev 2009;19: 353-364.

55 Deckersbach T, Nierenberg AA, Kessler R, Lund HG, Ametrano RM, Sachs G, et al: RESEARCH: cognitive rehabilitation for bipolar disorder: an open trial for employed patients with residual depressive symptoms. CNS NeurosciTher 2010;16:298-307.

56 Torrent C, del Mar Bonnin C, Martínez-Arán A, Valle J, Amann BL, González-Pinto A, et al: Efficacy of functional remediation in bipolar disorder: a multicenter randomized controlled study. Am J Psychiatry 2013;170:852859

57 Bowie CR, Gupta M, Holshausen K, Jokic R, Best M, Milev R: Cognitive remediation for treatment-resistant depression: effects on cognition and functioning and the role of online homework. J Nerv Ment Dis 2013;201: 680-685.

58 Calkins AW, McMorran KE, Siegle GJ, Otto MW: The effects of computerized cognitive control training on community adults with depressed mood. Behav Cogn Psychother 2015;43:578-589.

59 Elgamal S, McKINNON MC, Ramakrishnan K, Joffe RT, MacQUEEN G: Successful computer-assisted cognitive remediation therapy in patients with unipolar depression: a proof of principle study. Psychol Med 2007;37: 1229-1238

60 Lohman MC, Rebok GW, Spira AP, Parisi JM, Gross AL, Kueider AM: Depressive symptoms and memory performance among older adults rFrom the ACTIVE memory training intervention. J Aging Health 2013;25(8 suppl):209S-229S.

61 Mar1 L, Sotres JFC, León SO, Estrella J, Sosa JJS: Computer program in the treatment for major depression and cognitive impairment in university students. Comput Human Behav 2008;24:816-826.

62 Naismith SL, Diamond K, Carter PE, Norrie LM, Redoblado-Hodge MA, Lewis SJ, et al: Enhancing memory in late-life depression: the effects of a combined psychoeducation and cognitive training program. Am J Geriatr Psychiatry 2011;19:240-248.

63 Owens M, Koster EH, Derakshan N: Improving attention control in dysphoria through cognitive training: Transfer effects on working memory capacity and filtering efficiency. Psychophysiology 2013;50:297-307.

64 Segrave R, Arnold S, Hoy K, Fitzgerald P: Concurrent cognitive control training augments the antidepressant efficacy of tDCS: a pilot study. Brain Stimul 2014;7:325-331.

65 Siegle GJ, Price RB, Jones NP, Ghinassi F, Painter T, Thase ME: You gotta work at it pupillary indices of task focus are prognostic for response to a neurocognitive intervention for rumination in depression. Clin Psychol Sci 2014;2:455-471.

66 Meusel L-AC, Hall GB, Fougere P, McKinnon MC, MacQueen GM: Neural correlates of cognitive remediation in patients with mood disorders. Psychiatry Res Neuroimaging 2013;214:142-152.

67 Preiss M, Shatil E, Cermakova R, Cimermannova D, Flesher I: Personalized cognitive training in unipolar and bipolar disorder: a study of cognitive functioning. Front Human Neurosci 2013;7:108.

68 Motter JN, Pimontel MA, Rindskopf D, Devanand DP, Doraiswamy PM, Sneed JR: Computerized cognitive training and functional recovery in major depressive disorder: a metaanalysis. J Affect Disord 2016;189:184-191. 
69 Galderisi S, Piegari G, Mucci A, Acerra A, Luciano L, Rabasca AF, et al: Social skills and neurocognitive individualized training in schizophrenia: comparison with structured leisure activities. Eur Arch Psychiatry Clin Neurosci 2010;260:305-315.

70 Zhu X, Yin S, Lang M, He R, Li J: The more the better? A meta-analysis on effects of combined cognitive and physical intervention on cognition in healthy older adults. Ageing Res Rev 2016;31:67-79.

71 Smith PJ, Blumenthal JA, Hoffman BM, Cooper $\mathrm{H}$, Strauman TA, Welsh-Bohmer K, et al: Aerobic exercise and neurocognitive performance: a meta-analytic review of randomized controlled trials. Psychosomatic Med 2010; $72: 239$.

72 Zheng G, Xia R, Zhou W, Tao J, Chen L: Aerobic exercise ameliorates cognitive function in older adults with mild cognitive impairment: a systematic review and meta-analysis of randomised controlled trials. $\mathrm{Br} \mathrm{J}$ Sports Med 2016;50:1443-1450.

73 Groot C, Hooghiemstra A, Raijmakers P, van Berckel B, Scheltens P, Scherder E, et al: The effect of physical activity on cognitive function in patients with dementia: a meta-analysis of randomized control trials. Ageing Res Rev 2016;25:13-23.

74 Malchow B, Reich-Erkelenz D, OertelKnöchel V, Keller K, Hasan A, Schmitt A, et al: The effects of physical exercise in schizophrenia and affective disorders. Eur Arch Psychiatry Clin Neurosci 2013;263:451.

75 Barnes DE, Yaffe K: The projected effect of risk factor reduction on Alzheimer's disease prevalence. Lancet Neurol 2011;10:819-828.

76 Sofi F, Valecchi D, Bacci D, Abbate R, Gensini GF, Casini A, et al: Physical activity and risk of cognitive decline: a meta-analysis of prospective studies. J Int Med 2011;269:107-117.

77 Brondino N, Rocchetti M, Fusar-Poli L, Codrons E, Correale L, Vandoni M, et al: A systematic review of cognitive effects of exercise in depression. Acta Psychiatrica Scandinavica 2017;135:285-295.
78 Sharma V, Das S, Mondal S, Goswami U, Gandhi A: Effect of Sahaj Yoga on neuro-cognitive functions in patients suffering from major depression. Indian J Physiol Pharmacol 2006;50:375.

79 Kubesch S, Bretschneider V, Freudenmann R, Weidenhammer N, Lehmann M, Spitzer M, et al: Aerobic endurance exercise improves executive functions in depressed patients. J Clin Psychiatry 2003;64:1005-1012.

80 Oertel-Knöchel V, Mehler P, Thiel C, Steinbrecher K, Malchow B, Tesky V, et al: Effects of aerobic exercise on cognitive performance and individual psychopathology in depressive and schizophrenia patients. Eur Arch Psychiatry Clin Neurosci 2014;264:589.

81 Vasques PE, Moraes H, Silveira H, Deslandes AC, Laks J: Acute exercise improves cognition in the depressed elderly: the effect of dualtasks. Clinics 2011;66:1553-1557.

82 Bamidis P, Vivas A, Styliadis C, Frantzidis C Klados M, Schlee W, et al: A review of physical and cognitive interventions in aging. Neurosci Biobehav Revi 2014;44:206-220.

83 Gothe N, Pontifex MB, Hillman C, McAuley $\mathrm{E}$ : The acute effects of yoga on executive function. J Phys Act Health 2013;10:488-495.

84 Gothe NP, McAuley E: Yoga and cognition: a meta-analysis of chronic and acute effects. Psychosomatic Med 2015;77:784-797.

85 Deckersbach T, Hölzel BK, Eisner LR, Stange JP, Peckham AD, Dougherty DD, et al: Mindfulness-based cognitive therapy for nonremitted patients with bipolar disorder. CNS Neurosci Ther 2012;18:133-141.

86 Chiesa A, Calati R, Serretti A: Does mindfulness training improve cognitive abilities? A systematic review of neuropsychological findings. Clin Psychol Rev 2011;31:449-464.

87 Alfonso JP, Caracuel A, Delgado-Pastor LC, Verdejo-García A: Combined goal management training and mindfulness meditation improve executive functions and decisionmaking performance in abstinent polysubstance abusers. Drug Alcohol Depend 2011; 117:78-81.
88 Mitchell JT, Zylowska L, Kollins SH: Mindfulness meditation training for attention-deficit/ hyperactivity disorder in adulthood: current empirical support, treatment overview, and future directions. Cogn Behav Pract 2015;22: 172-191.

89 Soler J, Valdepérez A, Feliu-Soler A, Pascual JC, Portella MJ, Martín-Blanco A, et al: Effects of the dialectical behavioral therapy-mindfulness module on attention in patients with borderline personality disorder. Behav Res Ther 2012;50:150-157.

90 van der Velden AM, Kuyken W, Wattar U, Crane C, Pallesen KJ, Dahlgaard J, et al: A systematic review of mechanisms of change in mindfulness-based cognitive therapy in the treatment of recurrent major depressive disorder. Clin Psychol Rev 2015;37: 26-39.

91 Segal ZV, Williams JMG, Teasdale JD: Mindfulness-Based Cognitive Therapy for Depression: A New Approach to Relapse Prevention. New York, Guilford, 2002.

92 Kabat-Zinn J: Full Catastrophe Living: Using the Wisdom of Your Body and Mind in Everyday Life. New York, Delacorte, 1990.

93 Brunoni AR, Vanderhasselt MA: Working memory improvement with non-invasive brain stimulation of the dorsolateral prefrontal cortex: a systematic review and meta-analysis. Brain Cogn 2014;86:1-9.

94 Wolkenstein L, Plewnia C: Amelioration of cognitive control in depression by transcranial direct current stimulation. Biol Psychiatry 2013;73:646-651.

95 Escolano C, Navarro-Gil M, Garcia-Campayo J, Congedo M, De Ridder D, Minguez J: A controlled study on the cognitive effect of alpha neurofeedback training in patients with major depressive disorder. Front Behav Neurosci 2014;8:296.

96 Nuechterlein KH, Green MF, Kern RS, Baade LE, Barch DM, Cohen JD, et al: The MATRICS consensus cognitive battery, part 1: test selection, reliability, and validity. Am J Psychiatry $2008 ; 165: 203-213$. 